\title{
Cake Cutting Really is Not a Piece of Cake
}

\author{
Jeff Edmonds * $\quad$ Kirk Pruhs ${ }^{\dagger}$
}

\begin{abstract}
We consider the well-known cake cutting problem in which a protocol wants to divide a cake among $n \geq 2$ players in such a way that each player believes that they got a fair share. The standard RobertsonWebb model allows the protocol to make two types of queries, Evaluation and Cut, to the players. A deterministic divide-and-conquer protocol with complexity $O(n \log n)$ is known. Improving on previous lower bounds, we provide an $\Omega(n \log n)$ lower bound on the complexity of any deterministic protocol, even if the protocol is allowed to assign to a player a piece that is a union of intervals and only guarantee approximate fairness. We accomplish this by lower bounding the complexity to find for a single player, a piece of cake that is both rich in value, and thin in width. We then introduce a version of cake cutting in which the players are able to cut with only finite precision. In this case, we can extend the $\Omega(n \log n)$ lower bound to include randomized protocols.
\end{abstract}

\section{Introduction}

Our setting is a collection of self-interested entities who desire to partition a disparate collection of items of value. Imagine heirs of an estate wanting to divide the possessions of the newly departed. Or imagine the creditors of a bankrupt company, such as Enron, wanting to split up the company's remaining assets. The entities may well value the items differently. For example, one can imagine different heirs of an estate not necessarily agreeing on the relative value a baseball signed by Pete Rose, a worn leather lazy boy recliner, a mint condition classic Farrah Fawcett poster, etc. The goal is devise a protocol to split up the items fairly, that is, so every entity believes that he/she gets a fair share based on how he/she values the objects. Achieving this goal is potentially complicated by the fact that the entities may well be greedy, deceitful, treacherous, etc. They may not be honest about how they value the objects, they may collude together to cheat another entity, etc. So we seek a protocol that guarantees a fair share to everyone that is honest. If someone tries to cheat or lie, then they cannot blame the protocol if they don't end up with a fair share.

In the literature, this problem falls under the rubric of cake cutting $[1,6]$. (This is motivated by the well known phenomenon that some people value the frosting more than others.) The cake cutting problem arose from the 1940's school of Polish mathematicians. Since then the problem has blossomed and been widely popularized [6]. Most people find cake cutting problems psychologically and socially interesting, and some

\footnotetext{
*York University, Canada. jeff@cs.yorku.ca. Supported in part by NSERC Canada.

${ }^{\dagger}$ Computer Science Department. University of Pittsburgh. kirk@cs.pitt.edu. Supported in part by NSF grants CCR-0098752, ANI-0123705, CNS-0325353, and CCF-0448196.
} 
quick Googling reveals that cake cutting algorithms, and their analysis, are commonly covered by many SODA regulars in their algorithms and discrete mathematics courses.

Cake cutting in formalized in the following manner. The objects of value are ordered in some arbitrary way, and then abstracted away into subintervals of the interval $[0,1]$, which is the cake. Each entity/player has a value function $V$ that specifies how much that player values a particular subinterval, or more precisely, the objects in the subinterval. This is a reasonable model if the value of each item is small relative to the total value of the items. The protocol can query players about their value functions, which are initially unknown to the protocol. In the standard Robertson-Webb model [6, 7], the two types of queries are Evaluation and Cut. In an Evaluation query, a player is asked how much he values a subinterval. In a Cut query, the player is asked to identify an interval, with a fixed left endpoint, of a particular value.

\subsection{Previous Results}

Sgall and Woeginger [7] provide a nice brief overview of results in this area. Books by Robertson and Webb [6] and Brams and Taylor [1] provide more extensive overviews.

Let first consider upper bound results. A deterministic protocol that uses $\Theta\left(n^{2}\right)$ cuts was described in 1948 by Steinhaus in [8]. In 1984, Evan and Paz [2] gave a deterministic divide and conquer protocol that uses $\Theta(n \log n)$ cuts. Further, they gave a randomized protocol that uses $\Theta(n)$ cuts and $\Theta(n \log n)$ evaluations.

Approximately fair protocols were introduced by Roberson and Webb [5]. We say that a protocol is $c$-fair if it guarantees each honest player a piece of cake that he believes has value at least $\frac{1}{c n}$. There is a deterministic protocol that achieves $O(1)$-fairness with $\Theta(n)$ cuts and $\Theta\left(n^{2}\right)$ evaluations [5, 3, 9].

Traditionally, much of the research has focused on minimizing the number of cuts, without too much regard for the number of evaluations. In the settings that we are interested in, e.g. heirs splitting an inheritance, there is no good reason to assume that evaluation queries are especially easier or cheaper than cut queries. It is not clear why the initial focus was on minimizing cuts. One possibility is concern that too many cuts would lead to crumbling of a literal cake. In any case, we will view evaluation and cut queries as equally expensive.

Thus one can summarize the known upper bound results as follows. There is a deterministic protocol with complexity $O(n \log n)$ that guarantees exact fairness. No protocol that uses a linear number of queries is known, even if randomization is allowed, and even if the protocol need only guarantee $O(1)$-fairness.

So a natural avenue for investigation is to attempt to prove an $\Omega(n \log n)$ lower bound on the complexity of any cake cutting protocol. The most obvious way to prove such a lower bound is to try to reduce sorting (or more precisely, learning an unknown permutation) to cake cutting. A first step in this direction was taken by Magdon-Ismail, Busch, and Krishnamoothy [4], who were able to show that any protocol must make $\Omega(n \log n)$ comparisons to compute the assignment. So this result did not really lower bound the number of queries. A second step in this direction was taken by Sgall and Woeginger [7] who give a more complicated reduction from sorting to show an $\Omega(n \log n)$ lower bound on the complexity of any deterministic protocol that is required to assign to each player a piece that is a single subinterval of the cake. On the positive side, all known protocols have this property. On the other hand, there is no natural reason to impose this restriction in the settings that we are interested in. That is, it is perfectly reasonable to assign to an inheritor a collection of items that are not consecutive in the initial arbitrary ordering of the items. The lower bound of Sgall and Woeginger [7] can be seen to hold against randomized protocols. However, note that neither of these lower bounds $[4,7]$ hold if the protocol is only required to achieve approximate fairness. 


\subsection{Our Results}

In section 2, we give a lower bound of $\Omega(n \log n)$ on the complexity of any deterministic protocol for cake cutting, which is the first $\omega(n)$ lower bound in the general Robertson-Webb model. Our lower bound improves on the results in [7] in two ways: (1) it applies to protocols that may assign to a player a piece that is a union of intervals, and (2) it applies to protocols that only guarantee $n^{1-\delta}$ approximate fairness, that is, players can be allocated pieces with value as low as $\frac{1}{c n}=\frac{1}{n^{2-\delta}}$.

We believe that the main reasons why earlier lower bounds were not stronger is that they essentially attempted to reduce from sorting, which does seem to capture the difficulty of cake cutting in the general model. Instead, we observe that not only are the players required to find a piece that is rich in value, but if their pieces are not to overlap then most players need a piece that is thin in width. We obtain our $\Omega(n \log n)$ lower bound by showing a lower bound of $\Omega(\log n)$ on the complexity of a single player finding a piece that is both thin and rich, where thin means that the width at most $\frac{2}{n}$, and rich means that the value at least $\frac{1}{c n}$. It is easy to see how to find a piece that is thin and rich in $O(1)$ time using a randomized algorithm. With probability at least $\frac{1}{2}$, the interval $\left[C u t\left(0, \frac{i}{n}\right), C u t\left(0, \frac{i+1}{n}\right)\right]$ is thin and rich if $i$ is selected uniformly at random from $[0, n-1]$. Thus our deterministic lower bound does not extend to randomized algorithms.

To our knowledge, all the literature to date has assumed that players can answer cut and evaluation queries with exact precision. This is probably not so realistic in some settings, for example, it is probably too much to ask an inheritor to value an arbitrary subcollection of items to within a penny. For this reason, we introduce what we call approximate cut queries to which a player need only return an interval of cake of value within a $1+\epsilon$ factor of the requested value. To our knowledge, no one to date has considered approximate queries.

In section 3, we prove that if $\epsilon$ is a constant, then there is an $\Omega(n \log n)$ lower bound on the complexity of any randomized protocol for cake cutting with approximate cuts (with relative error $1+\epsilon$ ) and exact evaluation queries, even if only $n^{1-\delta}$-fairness is required. The fact that the protocol is allowed exact evaluations, but only approximate cuts, demonstrates the asymmetric power of these two operations. This lower bound is oblivious in that our adversary doesn't change the lower bound instance in response to random events internal to the protocol.

We believe that the main contribution of this paper, beyond the explicit lower bounds, is the identification of the importance of the problem of finding thin rich pieces. We also believe that the concept of approximate queries is interesting, and worthy of further investigation.

\subsection{Formal Problem Statement}

The cake consists of the interval $[0,1]$. Each player $p, 1 \leq p \leq n$, has value function $V_{p}\left(x_{1}, x_{2}\right)$ which specifies a value in the range $[0,1]$ that a player assigns to the subinterval $\left[x_{1}, x_{2}\right]$. Player values are scaled so that they each have value 1 for the whole cake, that is, $V_{p}(0,1)=1$. The value function should be additive, that is, $\forall x_{1} \leq x_{2} \leq x_{3} \in[0,1], V_{p}\left(x_{1}, x_{2}\right)+V_{p}\left(x_{2}, x_{3}\right)=V_{p}\left(x_{1}, x_{3}\right)$. In this paper, a piece of cake is a collection of subintervals, not necessarily a single subinterval. Further, the ends of each subinterval in a piece must have been at one of the ends of a cut. The value of a piece of cake is then just the sum of the values of the subintervals of the piece. The value functions are initially unknown to the protocol.

The protocol's goal is to assign to each player $p$ a piece $C_{p}$ of the cake. The pieces must be disjoint, that is, $C_{p}$ and $C_{q}$ must be disjoint for all players $p \neq q$. Further the protocol should be $c$-fair to each player $p$, that is, it must be the case that the value of $C_{p}$ according to $V_{p}$ is at least $\frac{c}{n}$. Thus one gets different variations to the problem depending on the value of $c$.

In order to achieve its goal in the Robertson and Webb model, the protocol may repeatedly ask any player one of two types of queries: 
- $\operatorname{AEval}_{p}\left(\epsilon, x_{1}, x_{2}\right)$ : This $1+\epsilon$ approximate evaluation query to player $p$ returns an $(1+\epsilon)$-approximate value of the interval $\left[x_{1}, x_{2}\right]$ of the cake for player $p$. That is, $\frac{1}{1+\epsilon} V_{p}\left(x_{1}, x_{2}\right) \leq \operatorname{AEval}_{p}\left(\epsilon, x_{1}, x_{2}\right) \leq$ $(1+\epsilon) V_{p}\left(x_{1}, x_{2}\right)$. An exact evaluation query, $\operatorname{Eval}_{p}\left(x_{1}, x_{2}\right)$, is equivalent to $\operatorname{AEval}_{p}\left(0, x_{1}, x_{2}\right)$.

- $\operatorname{ACut}_{p}\left(\epsilon, x_{1}, \alpha\right)$ : The $1+\epsilon$ approximate cut query returns an $x_{2} \geq x_{1}$ such that the interval of cake $\left[x_{1}, x_{2}\right]$ has value approximately $\alpha$ according to player $p$ 's value function $V_{p}$. More precisely, $x_{2}$ satisfies $\frac{1}{1+\epsilon} V_{p}\left(x_{1}, x_{2}\right) \leq \alpha \leq(1+\epsilon) V_{p}\left(x_{1}, x_{2}\right)$. An exact cut query, $C u t_{p}\left(\epsilon, x_{1}, \alpha\right)$, is equivalent to $\operatorname{ACut}_{p}\left(0, x_{1}, \alpha\right)$.

The protocol may be adaptive in the sense that the protocol need only decide on the $i$ th query after it has seen the outcome of the first $i-1$ queries and when randomized on coin flips. The complexity of a protocol is the worst-case, over all possible valuation functions, of the expected number of queries needed to accomplish its goal. For cake cutting, Las Vegas and Monte Carlo algorithms are of equal power; Since the complexity of verifying the correctness of an assignment has linear complexity, a Monte Carlo algorithm can be converted into a Las Vegas algorithm.

Sgall and Woeginger [7] point out, cut and evaluation queries can efficiently simulate all other types of queries used in protocols in the literature, e.g. cutting the cake into two parts with a specified ratio of value. There are many technical issues that must be considered when formally defining the "right" model. A nice discussion of these issues can be found in Sgall and Woeginger [7]. For example, in the standard model, after a cut, each piece is re-indexed to $[0,1]$. As we are proving lower bounds, it is more convenient to continue to index with respect to the entire cake. Several issues that are relevant when proving upper bounds - for example, further niceness properties on the value functions, and robustness against cheating are not particularly relevant to us here. Our value functions satisfy every niceness property considered in the literature. To prove our lower bound, it suffices to consider only the case when all players are honest. Our lower bounds are robust against reasonable minor modifications to the model.

\section{The Deterministic Lower Bound}

This section is devoted to proving the following theorem:

Theorem 1. The complexity of any deterministic protocol for cake cutting is $\Omega(n(\log n-\log c))$, even with exact queries and only c-approximate fairness is required. Note that this bound is $\Omega(n \log n)$ even when $c=n^{1-\delta}$.

We now consider a new game, that we call the thin-rich game, which takes place in the same setting as the cake cutting game. We then show that a bound of $\Omega(\log n-\log c)$ on the complexity of thin-rich will give a lower bound of $\Omega(n(\log n-\log c))$ for cake cutting.

Thin-Rich Game: This game involves a single player. We say that a piece of cake is thin if it has width at most $\frac{2}{n}$. We say a piece is rich if it has value at least $\frac{1}{c n}$ for this player. The goal for the protocol is to identify a thin rich piece of cake.

Lemma 2. If the deterministic complexity of thin-rich is $T(n)$ then the deterministic complexity for cake cutting game is $\Omega(n T(n))$.

Proof. In our model for the cake problem, it is equivalent to assume that each of the $n$ players is in a separate black box. The only interaction between them is via the sequence of queries given by protocol. Based on the previous answers proved by the players, the protocol chooses one player and either a evaluation or cut query for this player, to which the player responds. In the end, the protocol assigns each player a piece of 
the cake. Now consider this interaction from the perspective of one of the players. As far as he can tell, he is interacting with one deterministic protocol for the single player thin-rich game. From our assumption, if this player receives fewer than $T(n)$ queries, than there is a cake value distribution such that the piece of cake allocated to the player is not both thin and rich. If the cake cutting protocol makes fewer than $\frac{1}{2} n T(n)$ queries, then this is the case for more than half of the players. If any player fails to obtain a rich piece, the protocol fails. If more than half the players fail to obtain a thin piece, then the resulting pieces cannot be disjoint.

\subsection{Value Trees}

In this subsection we define what we call value trees and explain how a cake value distribution is derived from a value tree. Assume $n \geq 6$ is twice an integer power of 3 . The tree is a balanced 3-ary rooted tree with $\frac{n}{2}$ leaves, depth $L=\log _{3} \frac{n}{2}$, and a value $V(u)$ for each node. For each internal node $u$, its left, middle, and right children are denoted $l(u), m(u)$, and $r(u)$. Two of these three edges are labeled $\frac{1}{4}$ and are called light edges, and the remaining edge is labeled $\frac{1}{2}$ and is called a heavy edge. The value $V(u)$ of node is the product of the edge labels along the path from the root to $u$. Note that $u$ 's value is the sum of its children's values, i.e. $V(u)=\frac{1}{4} V(u)+\frac{1}{4} V(u)+\frac{1}{2} V(u)=V(l(u))+V(m(u))+V(r(u))$. Let $\ell(u)$ denote the number of edges in the path from the root to the node $u$. Let $q(u)$ denote the number of these that are heavy edges. It follows that $V(u)=\left(\frac{1}{2}\right)^{q(u)}\left(\frac{1}{4}\right)^{\ell(u)-q(u)}$.

The cake is partitioned into $\frac{n}{2}$ thin intervals, namely for $i \in\left[1, \frac{n}{2}\right]$, the $i$ th interval of width $\frac{2}{n}$ is $\left[\frac{2(i-1)}{n}, \frac{2 i}{n}\right]$. These $\frac{n}{2}$ intervals are associated with the leaves of the value tree. We associate with each internal node $u$, the interval of cake that is the union of the leaves of the subtree rooted at $u$. The width of this interval is $W(u)=3^{-\ell(u)}$, and its value is given by $V(u)$. If $u$ is a leaf, then this value is spread evenly over this interval.

The intuition may be useful. The canonical thin-rich piece, which is the goal of a protocol to find, consists a leaf $u$ with density $D(u)=\frac{V(u)}{W(u)}=\frac{1 / c n}{2 / n}=\frac{2}{c}$. Towards this goal, the protocol must find nodes in the tree that are both low in the tree and dense. In order for a node to have high density, the path from the root to it must have lots of heavy edges, namely

$$
D(u)=\frac{V(u)}{W(u)}=\frac{\left(\frac{1}{2}\right)^{q(u)}\left(\frac{1}{4}\right)^{\ell(u)-q(u)}}{\left(\frac{1}{3}\right)^{\ell(u)}} \geq \frac{2}{c}
$$

Or equivalently,

$$
q(u) \geq \log _{2}\left(\frac{4}{3}\right) \ell(u)-\log _{2} c>\frac{4}{10} \ell(u)-\log _{2} c
$$

The obvious $O(\log n)$ time protocol, starts at the root, which has density $D(u)=1$, and follows the unique path consisting of only heavy edges down the tree. If a deterministic protocol attempts to circumvent this process by leaping to a lower node, then the adversary can simply fix the edges in the path to this node to be light. If a randomized protocol selects a random node, then each edge is heavy with probability $\frac{1}{3}$ giving $q(u)=\frac{1}{3} \ell(u)$, which is much less than the $q(u)=\frac{4}{10} \ell(u)-\log _{2} c$ needed for it to be rich.

We say that a protocol for the thin-rich game is normal if, when the input value distribution is derived from a value tree, the protocol always returns a leaf of the value tree. We now show that, without loss of generality, we may restrict our attention to normal protocols.

Lemma 3. If there is a deterministic protocol A for thin-rich with complexity $T(n)$, then for value distributions derived from value trees, there is a normal deterministic protocol $B$ with complexity $O(T(n))$.

Proof. Consider an arbitrary protocol $A$. Let $\mathcal{I}$ denote the collection of intervals returned by $A$. Because overall $\mathcal{I}$ has density at least $\frac{2}{c}$, at least one $I$ of these intervals in $\mathcal{I}$ does as well. Since the cardinality of 
$\mathcal{I}$ is at most $T(n)$, one such interval $I$ can be found in time $O(T(n))$. Because this interval has width at most $\frac{2}{n}$, it overlaps with at most two leaves of the value tree. Because each leaf has uniform value along its width, at least one of these two leaves must have density at least $\frac{2}{c}$. The protocol must know the value of each of the intervals in $\mathcal{I}$ (or as subinterval) or else this interval might have no value. Hence, with at most one additional evaluation, the protocol has enough information to find this leaf.

As a protocol for thin-rich asks queries, it gains information about the value tree. In order to bound the information learned, the lower bound adversary reveals the labels of enough edges of the value tree to provide the protocol with at least as much information as the thin-rich protocol gets from the query. Let $P=u_{0}, \ldots, u_{k}$ be a path from the root $u_{0}$ of the value tree to a node $u_{k}$. The node $u_{k}$ is said to be revealed if all the labels on all the edges leading from a node $u_{i}, 0 \leq i \leq k-1$, to a child or $u_{i}$, are revealed. Lemma 4 quantifies what can be learned from revealed vertices.

\section{Lemma 4.}

- For any revealed node $u$, the value $V(u)$ of the interval of cake under it can be computed.

- Let $u$ be a revealed node, let $x$ be the leftmost point in $u$, and $y$ the rightmost point in $u$. Then $V(0, x)$ and $V(0, y)$ can be computed.

- Let $x$ be a point in a revealed leaf $u$, and let $y \geq x$ be a point in a revealed leaf $v$. Then $V(0, x)$ and $V(x, y)$ can be computed.

- Let $u$ be a revealed leaf, let $x_{1}$ is a point in $u$, and let $\alpha$ be a cake value. From this information, the least common ancestor of $u$ and the node $v$ that contains the point $x_{2}$ satisfying $V\left(x_{1}, x_{2}\right)=\alpha$ can be computed.

Proof. We consider the items one by one. For the first item, $V(u)$ is just the product of the edge labels leading to $u$. Consider the second item. Let $u_{0}, \ldots, u=u_{k}$ be the path from the root to $u$. $V(0, x)$ is then just the sum of the values of the siblings to the left of a $u_{i}, 1 \leq i \leq k$, which may be computed by the previous item. $V(0, y)$ is then $V(0, x)+V(u)$. Consider the third item. Let $x^{\prime}$ be the left most point in the leaf $u$. Because the value of the cake is uniform on leaves, $V\left(x^{\prime}, x\right)=\frac{x-x^{\prime}}{2 / n} \cdot V(u)$. Then $V(0, x)=$ $V\left(0, x^{\prime}\right)+V\left(x^{\prime}, x\right)$ and $V(x, y)=V(0, y)-V(0, x)$. Consider the fourth item. Let $u_{0}, \ldots, u_{L}=u$ be the path from the root to the leaf $u$ containing $x_{1}$. Proceed up the tree from $u$, computing $V\left(x_{1}, y_{i}\right)$ where $y_{i}$ is the right most point under $u_{i}$. Here $V\left(x_{1}, y_{L}\right)=\frac{y_{L}-x_{1}}{2 / n} \cdot V(u)$ and $V\left(x_{1}, y_{i}\right)$ is $V\left(x_{1}, y_{i+1}\right)$ plus the sum the values of the children of $u_{i}$ that are to the right of $u_{i+1}$. When the sum exceeds $\alpha$, then $u_{i}$ is the least common ancestor.

Lemma 5. The deterministic complexity of thin-rich is $\Omega(\log n-\log c)$.

Proof. We lower bound the complexity of a normal protocol $A$ on a value tree distribution. We maintain a number of invariants. First, is that the protocol $A$, knows nothing about the value tree except for the edges that have been revealed by the adversary. Second, for each node, either none or all three of its outgoing edges have been revealed. Third, at each point in time the set of revealed nodes forms of a connected component of the value tree that contains the root. Finally, after $k$ queries, any root to leaf path contains at most $2 k$ edges revealed to be heavy. Initially, these invariants are trivially true.

Suppose that on the $k^{t h}$ query, the protocol $A$ makes the query $\operatorname{Eval}\left(x_{1}, x_{2}\right)$. Let $u_{0}, \ldots u_{L}$ be the path from the root to the leaf containing $x_{1}$. Let $u_{i}$ be the lowest revealed node in this path. The edges in $u_{i}, \ldots u_{L}$ are revealed to be light. For each of these nodes, when its outgoing edge is revealed to be light, one of its other two outgoing edges is reveal to be light and the other heavy. Note that this automatically 
reveals not only all the nodes in this path, but also reveals all the children of the nodes in this path. This same process is then repeated for $x_{2}$. By Lemma 4, $A$ will then have enough information to compute the answer to this Eval query. It is easy to verify that the invariants are maintained.

Now suppose that on the $k^{t h}$ query, the protocol $A$ makes the query $C u t\left(x_{1}, \alpha\right)$. As done for an Eval query, the "forked" path from the root to the leaf containing $x_{1}$ is revealed. As given by Lemma 4, let $u_{i}$ be the least common ancestor of the leaf containing $x_{1}$ and the leaf containing the unknown point $x_{2}$ satisfying $V\left(x_{1}, x_{2}\right)=\alpha$. We will now describe how to recursively determine and reveal the path $U$ from $u_{i}$ to $x_{2}$ in such a way that all these edges on this path are light. Let $v$ denote the first unrevealed node in $U$. Let $\beta$ be the value of cake that $A$ seeks from the subtree rooted at $v$. This is well defined by Lemma 4 . If $\frac{\beta}{V(v)} \leq \frac{1}{2}$, then the three edges leading to children of $v$ are labeled $\left[\frac{1}{4}, \frac{1}{4}, \frac{1}{2}\right]$, otherwise, they are labeled $\left[\frac{1}{2}, \frac{1}{4}, \frac{1}{4}\right]$. Note that either way, the next edge in $U$ will be a light edge. This process ends when $v$ becomes the leaf containing $x_{2}$. By Lemma 4, $A$ now has enough information to determine the value of $x_{2}$. It is easy to verify that all invariants are maintained.

Suppose that the protocol terminates after $\left(\frac{4}{10} L-\log _{2} c\right) / 2$ queries claiming that a leaf node $u$ is rich. The second invariant states that at most $\frac{4}{10} L-\log _{2} c$ edges on the path from the root to $u$ have been revealed to be rich. By making the rest of the edges in this path light, we can make $u$ not rich. This then contradicts the correctness of $A$.

\section{The Randomized Lower Bound}

This section is devoted to proving the following theorem.

Theorem 6. If a protocol can only make $1+\epsilon$ approximate queries, and c-fairness is required, then the complexity of any randomized protocol for cake cutting is $\Omega\left(n \log \frac{n}{c} / \log \frac{1}{\epsilon}\right)$.

Our proof uses Yao's technique, which states that it is sufficient to exhibit an input distribution on which the average-case time of every deterministic protocol is $\Omega\left(n \log \frac{n}{c} / \log \frac{1}{\epsilon}\right)$. Our input distribution, chooses independently for each player a random value tree from which to derive his value distribution. This is done by choosing independently for each node in the tree, one of its outgoing edges to be heavy. We again reduce cake cutting to the thin-rich game.

Lemma 7. Assume that any protocol for the thin-rich game that makes fewer than $T(n)$ queries fails to obtain a thin-rich piece with probability at least $\frac{3}{4}$ when given a random value distribution. If follows that any protocol for cake cutting that makes fewer than $\frac{1}{4} n T(n)$ queries fails with high probability when the players are given independently chosen value distributions.

Proof. As we did in the proof of Lemma 2, assume that each player is a separate black box. From our assumption, if this player receives fewer than $T(n)$ queries, then he fails to obtain a thin-rich piece with probability $\frac{3}{4}$. If the cake cutting protocol makes fewer than $\frac{1}{4} n T(n)$ queries, then this is the case for more than $\frac{3}{4} n$ of the players. Hence, the expected number of players that do not obtain a thin-rich piece is at least $\frac{9}{16} n$ and because these events are independent, with high probability more than half the players fail. As done before, this means that the players pieces cannot be rich and non-overlapping.

\subsection{The Path and Triangle Game}

As in the deterministic lower bound, we may restrict our attention to normal thin-rich protocols, that is, those that return a leaf in the value tree. We now introduce a game, the path and triangle game, that we show 
captures the complexity of finding path in the value tree that is sufficiently rich in heavy edges to give a rich leaf.

Definition of the Path and Triangle Game: The protocol is given a value tree, except that it does not know the value of the labels. The protocol makes a sequence of queries, where each query is either a path query or a triangle query. Both types of queries specify a node $u$ in the tree. In response to a path query, the labels on all of the edges incident to a node on the path from the root to $u$ are revealed to the protocol. In response to a triangle query, the labels on all the edges, on all the paths leading from $u$ to descendants of $u$, up to depth $\gamma=2+\lg \left(\frac{1}{\epsilon}\right)$, in the subtree rooted at $u$, are revealed to the protocol. The protocol's goal is to find a rich path, i.e. one with at least $\frac{4}{10} L-\log _{2} c$ heavy edges. The complexity of a particular protocol is the number of path and triangle queries needed to accomplish this goal.

\subsection{From Thin-Rich to Path and Triangle}

We now show how to reduce the thin-rich game to the path and triangle game.

Lemma 8. If the complexity of the path and triangle game is lower bounded by $T(n)$ for a random value tree, then the complexity of thin-rich game is $\Omega(T(n))$ when the value distribution is derived from a random value tree.

Proof. We will prove the contrapositive, that is, a thin-rich protocol $A$ with complexity $T(n)$ implies the existence of a protocol $B$ for the the path and triangle game with complexity $O(T(n))$. We construct $B$ by simulating $A$.

Suppose that protocol $A$ makes the query $\operatorname{Eval}\left(x_{1}, x_{2}\right)$. Protocol $B$ then makes two path queries: one query to the leaf containing $x_{1}$ and one query to the leaf containing $x_{2}$. The value $V\left(x_{1}, x_{2}\right)$ can then be computed by Lemma 4, and is then returned to $A$ as the result to the Eval query.

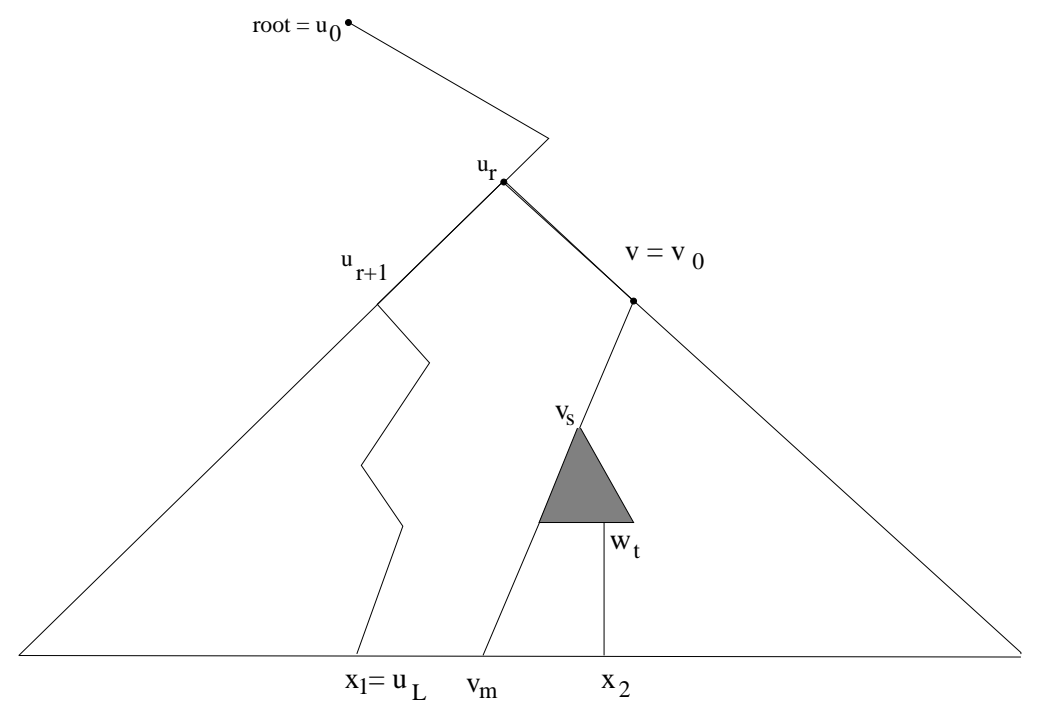

Figure 1: The two path queries and one triangle query associated with an approximate cut

Suppose that protocol $A$ makes the query $\operatorname{ACut}\left(\epsilon, x_{1}, \alpha\right)$. Let $x_{2}$ denote the point that $A$ seeks, that is the point such that $V\left(x_{1}, x_{2}\right)=\alpha$. Note that at this point in time, neither $A$ nor $B$ may know the exact value of $x_{2}$, but nevertheless we wish to reason about $x_{2}$. Protocol $B$ then makes at most two path queries and at most one triangle query. After these queries, protocol $B$ will have enough information to provide protocol 
$A$ with a point $y$ such that $V\left(x_{1}, y\right)$ is sufficiently close to $\alpha$. We now define these three queries. Figure 1 may be useful in understanding the queries.

The First Path Query: Let $u_{0}, u_{1}, \ldots, u_{L}$ be the sequence of nodes along the path from the root to the leaf containing $x_{1}$ in the tree. The first path query is to the node $u_{L}$. If both $x_{1}$ and $x_{2}$ are in $u_{L}$ then $B$ may return $x_{2}$. If $x_{2}$ is not in the leaf $u_{L}$, then $B$ makes a second path query.

The Second Path Query: Using Lemma 4, Protocol $B$ computes the least common ancestor $u_{r}$ of the leaf containing $x_{1}$ and the leaf containing $x_{2}$. All of the children of $u_{r}$ must be revealed since $u_{r+1}$ is on the revealed path from the root to $x_{1}$. Let $v$ be the child of $u_{r}$ containing $x_{2}$. The second path query is to the leftmost leaf $v_{m}$ in the subtree rooted at $v$. Let $v=v_{0}, v_{1}, \ldots v_{m}$ be the nodes along the path from $v$ to $v_{m}$

The Triangle Query: Again using Lemma 4, Protocol $B$ computes the least common ancestor $v_{s}$ of $v_{m}$ and the leaf containing $x_{2}$. The triangle query is to the node $v_{s}$.

Computing the Result: If the height of $v_{s} \leq \gamma$ then the leaf containing $x_{2}$ is known to $B$. The value of $x_{2}$ can then can be computed by Lemma 4 and is returned to protocol $A$. Otherwise, let $w_{1}, w_{2}, \ldots, w_{2^{\gamma}}$ be the descendants of $v_{s}$ of depth $\gamma$ in the subtree rooted at $v_{s}$. Let $w_{t}$ be the node such that $x_{2}$ is in the subtree rooted at $w_{t}$. The point $y$ returned by protocol $B$ will any point in the interval $w_{t}$.

We now argue the correctness of the result returned by protocol $B$. Because both $x_{2}$ and $y$ are under $w_{t}$, the error $V\left(x_{1}, y\right)-\alpha$ will be at most $V\left(w_{t}\right)$. We have $V\left(w_{t}\right) \leq\left(\frac{1}{2}\right)^{\gamma} V\left(v_{s}\right)$, since $w_{t}$ is $\gamma$ edges below $v_{s}$ and every edge has a label of at most $\frac{1}{2}$. We have $V\left(v_{s}\right) \leq 4 V\left(l\left(v_{2} s\right)\right.$, because the edge to the left child $l\left(v_{s}\right)$ of $v_{s}$ has a label of at least $\frac{1}{4}$. We have $4\left(\frac{1}{2}\right)^{\gamma} \leq \epsilon$, by the definition of $\gamma=2+\lg \left(\frac{1}{\epsilon}\right)$. We have $V\left(l\left(v_{2} s\right) \leq \alpha\right.$, since the interval under $l\left(v_{s}\right)$ is totally contained in the interval of value $\alpha$ to the right of $x_{1}$. Combining these gives that $V\left(w_{t}\right) \leq \epsilon \alpha$. This completes simulation of the $A C u t\left(\epsilon, x_{1}, \alpha\right)$ query.

In the end, protocol $A$ finds a rich leaf, which provides protocol $B$ with a rich path.

\subsection{The Analysis of the Path and Triangle Game}

This section is devoted to proving that with probability $\Omega(1)$ the complexity of every randomized protocol for the path and triangle game is $\Omega\left(\log \frac{n}{c} / \log \frac{1}{\epsilon}\right)$ if the input is a random value tree. Let Det be the set of nodes $u$ which have been revealed, i.e. labels on path to $u$ are known. We define a potential function $F(u)$ on a node $u$ by $\left(\frac{11}{4} q(u)-\ell(u)\right)$. Note that for a random node, $q(u)=\frac{1}{3} \ell(u)$ and $F(u)=\frac{11}{4} \frac{1}{3} \ell(u)-\ell(u)=$ $-\frac{1}{12} \ell(u)$, but for a rich path $q(u) \approx \frac{4}{10} \ell(u)$ and $F(u) \approx \frac{11}{4} \frac{4}{10} L-L=\frac{1}{10} L$. We define a potential function $F$ for the state of the game by $F=\max _{u \in D e t} F(u)$. Initially Det consists of only the root. As $\ell($ root $)=0$, and $q($ root $)=0$, it is the case that initially $F=0$. We now bound the expected change in the value of the potential function as the result of a single query.

Lemma 9. There exists a constant $\beta$, such that the expected change in $F$ as the result of one query is at most $2 \gamma+\beta$.

Proof. First consider the path operation. The player specifies one leaf $x$ and learns the labels on the path $U$ from the root to $x$ (plus the labels on the other edges that lead from a node $y$ on $U$ to a child of $y$ ). Let $u$ be the last node in $U$ that was in Det before the path query. Let $v$ be the node for which $F$ is maximized after the path query. When $F$ changes, there are two cases. First assume that $v$ is in on the path $U$. Let $\ell^{\prime}$ be the number of edges from $u$ to $v$ and $q^{\prime}$ be the number of these which are heavy. Hence $F^{\prime}$, the amount that $F$ increases by, is $\frac{11 q^{\prime}}{4}-\ell^{\prime}$. Note that $F^{\prime} \geq f$ is equivalent to $q^{\prime} \geq \frac{4 f^{\prime}}{11}+\frac{4 l^{\prime}}{11}$. We then use this to bound the 
expected value of $F^{\prime}$.

$$
\begin{aligned}
E\left[F^{\prime}\right] & =\int_{f \geq 0} f \cdot \operatorname{Pr}\left[F^{\prime}=f\right] \\
& =\int_{f \geq 0} \operatorname{Pr}\left[F^{\prime} \geq f\right] \\
& \leq \int_{f \geq 0} \sum_{m \geq f} \operatorname{Pr}\left[\ell^{\prime}=m \text { and } q^{\prime} \geq \frac{4 f^{\prime}}{11}+\frac{4 m}{11}\right] \\
& =\int_{f \geq 0} \sum_{m \geq f} \operatorname{Pr}\left[\ell^{\prime}=m \text { and } q^{\prime} \geq\left(1+\left(\frac{12 f}{11 m}+\frac{1}{11}\right)\right) \frac{m}{3}\right]
\end{aligned}
$$

If $\ell^{\prime}$ is fixed to be $m$, then $q^{\prime}$ is binomially distributed with mean $\frac{m}{3}$. Using a Chernoff bounds we know that $\operatorname{Pr}\left[q^{\prime} \geq(1+\delta) \frac{m}{3}\right] \leq e^{-\delta^{2} m / 6}$. In our case, $\delta=\left(\frac{12 f}{11 m}+\frac{1}{11}\right)$. Hence,

$$
\begin{aligned}
E\left[F^{\prime}\right] & \leq \int_{f \geq 0} \sum_{m \geq f} \exp \left(-\left(\frac{12 f}{11 m}+\frac{1}{11}\right)^{2} \cdot \frac{m}{6}\right) \\
& =\int_{f \geq 0} \sum_{m \geq f} \exp \left(\frac{-24 f^{2}}{121 m}\right) \cdot \exp \left(\frac{-4 f}{121}\right) \cdot \exp \left(\frac{-m}{726}\right) \\
& =\sum_{m \geq 0} \exp \left(\frac{-m}{726}\right) \int_{0 \leq f \leq m} \exp \left(\frac{-24 f^{2}}{121 m}\right) \cdot \exp \left(\frac{-4 f}{121}\right) \\
& =\sum_{m \geq 0} \exp \left(\frac{-m}{726}\right) \cdot O\left(\exp \left(\frac{-24}{121 m}\right)\right) \\
& =\sum_{m \geq 0} \exp \left(\frac{-m}{726}\right) \cdot O(1) \\
& =O(1)
\end{aligned}
$$

This completes that case when the node $v$ for which $F$ is maximized is in on the path $U$. The only remaining case is when $v$ is a child of a node on the path $U$. For such nodes, $q(v)$ can be at most one more than the value of $F$ on $v$ 's parent. Thus the expected change of $F$ of siblings of nodes in $U$ is at most an additive constant more than the expected change on the nodes in $U$.

Now consider a triangle operation to a node $u$. The protocols learns all the labels to a depth $\gamma$ below $u$. For any node $v$, this increases $q(v)$ by at most $\gamma$. The increase in $\ell(v)$ has to be at least the increase of $q(v)$. Thus $F$ can increase by at most $\left(\frac{11 \gamma}{4}-\gamma\right) \leq 2 \gamma$.

We are now ready to establish the lower bound for the path and triangle game.

Lemma 10. Any protocol for the path and triangle game that makes fewer than $T(n)=\Omega\left(\log \frac{n}{c} / \log \frac{1}{\epsilon}\right)$ queries fails with probability at least $\frac{3}{4}$ to find a rich path.

Proof. Finding a rich path involves finding a leaf $u$ with $q(u) \geq\left(\frac{4}{10} L-\lg 2 c\right), l(u)=L$, and $F(u)=$ $\frac{11}{4} q(u)-l(u) \geq \frac{11}{4}\left(\frac{4}{10} L-\lg 2 c\right)-L=\left(\frac{1}{10} L-\frac{11}{4} \lg 2 c\right)$. However, Lemma 9 proves that at each time step, the expected change in $F$, is at most $2 \gamma+\beta$. Therefore, after fewer than $T(n)$ queries, $E[F]$, the expected value of $F$, is at most $(2 \gamma+\beta) T(n)$. By Markov's inequality, the probability that $F \geq 4 E[F]$ is at 
most $\frac{1}{4}$. Hence, setting $T(n)=\frac{1}{4(2 \gamma+\beta)}\left(\frac{1}{10} L-\frac{11}{4} \lg 2 c\right)$ gives a contradiction. Plugging in $\gamma=2+\lg \left(\frac{1}{\epsilon}\right)$ and $\beta=O(1)$ gives $T(n)=\Omega\left(\log \frac{n}{c} / \log \frac{1}{\epsilon}\right)$ as required.

We finish with a few comments on the tightness of our lower bounds with approximate queries. If exact queries are replaced by $1+\epsilon$-approximate queries, then the $\Theta(n \log n)$ time divide and conquer protocol returns only $(1+\epsilon)^{\log (n)}$-fair pieces, because the error accumulates multiplicatively at each of the $\log (n)$ levels of recursion. Doing the same for the $\Theta\left(n^{2}\right)$ time protocol introduces only $1+\epsilon$ error to the final fairness. If the model allows only $1+\epsilon$-approximate queries, for some constant $\epsilon$, but requires only $(1+\epsilon)^{\log (n)}<<$ $n^{1-\delta}$-fairness, then our lower bound of $\Omega(n \log n)$ is tight. If the model allows only $1+\frac{1}{\log n}$-approximate queries and requires $O(1)$-fairness, then our lower bound of $\Omega(n \log n \log \log n)$ is off by at most a $\log \log n$ term. The outstanding open question in this area is the exact complexity of achieving $O(1)$-fairness with $O(1+\epsilon)$-approximate queires.

\section{References}

[1] S.J. BRAms AND A.D. TAYLOR (1996). Fair Division - From cake cutting to dispute resolution. Cambridge University Press, Cambridge.

[2] S. Even And A. PaZ (1984). A note on cake cutting. Discrete Applied Mathematics 7, 285-296.

[3] S.O. Krumke, M. Lipmann, W. de Paepe, D. Poensgen, J. Rambau, L. Stougie, and G.J. WoEginger (2002). How to cut a cake almost fairly. Proceedings of the 13th Annual ACM-SIAM Symposium on Discrete Algorithms (SODA’2002), 263-264.

[4] M. Magdon-Ismail, C. Busch, and M.S. Krishnamoorthy (2003). Cake cutting is not a piece of cake. Proceedings of the 20th Annual Symposium on Theoretical Aspects of Computer Science (STACS'2003), LNCS 2607, Springer Verlag, 596-607.

[5] J.M. Robertson AND W.A. WebB (1995). Approximating fair division with a limited number of cuts. Journal of Combinatorial Theory, Series A 72, 340-344.

[6] J.M. RoberTSON AND W.A. WebB (1998). Cake-cutting algorithms: Be fair if you can. A.K. Peters Ltd.

[7] J. Sgall And G. J. Woeginger (2003). A lower bound for cake cutting. LNCS 2461, Springer Verlag, 896-901. Proc. of the 11th Ann. European Symp. on Algorithms (ESA), Lecture Notes in Comput. Sci. 2832, Springer, 459-469.

[8] H. Steinhaus (1948). The problem of fair division. Econometrica 16, 101-104.

[9] G.J. Woeginger (2002). An approximation scheme for cake division with a linear number of cuts. Proc. of the 10th Ann. European Symp. on Algorithms (ESA), Lecture Notes in Comput. Sci. 2461, Springer, 896-901. 